\title{
BMJ Open Comparison of a single high-sensitivity cardiac troponin $T$ measurement with the HEART score for rapid rule-out of acute myocardial infarction in a primary care emergency setting: a cohort study
}

To cite: Johannessen TR, Atar D, Vallersnes 0M, et al. Comparison of a single highsensitivity cardiac troponin $\mathrm{T}$ measurement with the HEART score for rapid rule-out of acute myocardial infarction in a primary care emergency setting: a cohort study. BMJ Open 2021;11:e046024. doi:10.1136/ bmjopen-2020-046024

- Prepublication history and additional material for this paper are available online. To view these files, please visit the journal online (http://dx.doi. org/10.1136/bmjopen-2020046024).

Received 19 0ctober 2020 Revised 28 January 2021 Accepted 04 February 2021
Check for updates

(C) Author(s) (or their employer(s)) 2021. Re-use permitted under CC BY-NC. No commercial re-use. See rights and permissions. Published by BMJ.

For numbered affiliations see end of article.

Correspondence to

Dr Tonje R Johannessen;

t.r.johannessen@medisin.uio.no

\section{ABSTRACT}

Objective This study aims to compare the rule-out safety of a single high-sensitivity cardiac troponin $T$ (hs-cTnT) with the History, ECG, Age, Risk factors and Troponin (HEART) score in a low-prevalence primary care setting of acute myocardial infarction (AMI). Participants Patients with non-specific symptoms suggestive of AMI were consecutively enroled at a primary care emergency clinic in 0slo, Norway from November 2016 to October 2018.

Methods After initial assessment by a general practitioner, hs-cTnT samples were drawn. AMI was ruled-out by a single hs-cTnT $<5 \mathrm{ng} / \mathrm{L}$ measured $\geq 3$ hours after symptom onset. The HEART score was calculated retrospectively; a score $\leq 3$ of 10 points was considered low risk. We also calculated a modified HEART score using more sensitive hs-cTnT thresholds. The primary outcome was the diagnostic performance for the rule-out of AMI at the index event; the secondary the composite of AMI or all-cause death at 90 days. Results Among 1711 patients, 61 (3.6\%) were diagnosed with $\mathrm{AMI}$, and 569 (33.3\%) patients were assigned to single rule-out $(<5 \mathrm{ng} / \mathrm{L})$. With no AMls in this group, the negative predictive value (NPV) and sensitivity were both $100.0 \%(95 \% \mathrm{Cl} 99.4 \%$ to $100.0 \%$ and $94.1 \%$ to $100.0 \%$, respectively), and the specificity $34.5 \%$ ( $32.2 \%$ to $36.8 \%)$. The original HEART score triaged more patients as low risk $(n=871)$, but missed five AMls (NPV 99.4\% (98.7\% to 99.8\%); sensitivity $91.8 \%(81.9 \%$ to $97.3 \%)$ and specificity $52.5 \%$ (50.0\% to $54.9 \%)$ ). The modified HEART score increased the low-risk sensitivity to $98.4 \%$ (91.2\% to $100.0 \%)$, with specificity $38.7 \%$ (36.3\% to $41.1 \%$ ). The 90 -day incidence of AMI or death in the single rule-out and the original and modified low-risk HEART groups were $0.0 \%$, $0.7 \%$, and $0.2 \%$, respectively.

Conclusion In a primary care emergency setting, a single hs-cTnT strategy was superior to the HEART score in ruling out AMI. This rapid and safe approach may enhance the assessment of patients with chest pain outside of hospitals.

Trial registration number NCT02983123.

\section{Strengths and limitations of this study}

- The diagnostic ability to rule-out acute myocardial infarction by a single high-sensitivity cardiac troponin $\mathrm{T}$ was investigated and compared with the History, ECG, Age, Risk factors and Troponin (HEART) score in a primary care population.

- The observational cohort comprised a low-risk population enroled in a primary care emergency setting with few missing data.

- The study was embedded in the daily routine at the clinic, reducing bias and increasing the internal validity of the results.

- The study may not be adequately powered, as the total number of events was low.

The HEART score was calculated retrospectively.

\section{INTRODUCTION}

Non-ST-segment elevation acute coronary syndrome (NSTE-ACS) is an important differential diagnosis in patients presenting with acute chest pain in primary care. ${ }^{12}$ Patients with chest pain or other symptoms suggestive of NSTE-ACS are often admitted to hospitals for further examination due to limited reliable diagnostic decision tools. ${ }^{3-5}$

The last decade has seen an increased focus on the diagnostic assessment with troponins outside of hospitals. Point-of-care (POC) troponin assays, used in general practice ${ }^{67}$ or by emergency medical services (EMS), ${ }^{89}$ are useful in identifying high-risk patients. Still, they may not be sufficiently safe to rule-out acute myocardial infarction (AMI) ${ }^{9}{ }^{10}$ The History, ECG, Age, Risk factors and Troponin (HEART) score,${ }^{11} 12$ initially developed for the emergency departments (EDs), has proven to be a valuable decision aid for the EMS, identifying low-risk and high-risk patients in the prehospital setting. ${ }^{13}$ The diagnostic 
parameters of the HEART score were improved when the conventional troponin assay was replaced with a high-sensitivity cardiac troponin (hs-cTn) assay. ${ }^{15} 16 \mathrm{In}$ addition, high diagnostic performance has recently been demonstrated for novel hs-cTn POC assays in hospital cohorts, but these are not yet validated for primary care. ${ }^{17} 18$ Therefore, a strategy that safely excludes AMI outside the hospital ED is still needed. ${ }^{10} 14$

We recently found a high rule-out safety for AMI in a primary care emergency setting using the European Society of Cardiology's (ESC) $0 / 1$ hour algorithm for hs-cTnT. ${ }^{19}$ As serial hs-cTnT measurements represent a logistic challenge in many primary care settings, ${ }^{20}$ it was of interest to investigate the diagnostic and prognostic performance of a single hs-cTnT measurement. For the hospital setting, high rule-out safety has been demonstrated for an undetectable $(<5 \mathrm{ng} / \mathrm{L})$ hs-cTn measurement in patients presenting to the ED more than 3 hours after symptom onset. ${ }^{21}{ }^{22}$ However, evidence of the safety of a single hs-cTnT rule-out approach remains sparse for the primary care setting. Further, to the best of our knowledge, the single hs-cTnT rule-out approach has not yet been validated and compared with the HEART score in a primary care emergency setting, where patients have a low pretest probability for acute coronary syndrome (ACS).

The aim of this study was, therefore, to investigate whether the single hs-cTnT strategy was safe to rule-out AMI in patients presenting with non-specific symptoms in a primary care emergency setting, and compare it with the HEART score.

\section{METHODS}

\section{Study design and population}

This study was a planned secondary analysis of the prospective, observational One hoUr Troponin in a lowprevalence population of Acute Coronary Syndrome (OUT-ACS) study $^{19}$ conducted from November 2016 to October 2018 at the Oslo Accident and Emergency Outpatient Clinic (OAEOC); the main primary care emergency clinic in Oslo, Norway.

The OAEOC is staffed by general practitioners (GPs) and nurses and offers serial hs-cTnT sampling to rule-out AMI at the OAEOC observation unit 24 hours a day, all year. Patients (18 years and older) with acute nontraumatic chest pain or other non-specific symptoms admitted at the clinic for hs-cTnT, were consecutively enroled. Patients with potential atypical AMI presentation, for example, acute dyspnoea, acute fatigue or diaphoresis, were also eligible. In cases of a highly suspected ACS, including ST-segment elevation myocardial infarction, patients were directly hospitalised and not available for study enrolment. Patients with chronic kidney disease (estimated glomerular filtration rate of $<30 \mathrm{~mL} / \mathrm{min} / 1.73 \mathrm{~m}^{2}$ ) were excluded (figure 1). Further details regarding the study setting and participants have been described previously. ${ }^{19}$
Clinical assessment and measurement of hs-cTnT

Medical history, physical examination, pulse oximetry and a 12-lead ECG were obtained by the GP for all patients presenting to the OAEOC with symptoms suggestive of ACS. If indicated, chest X-ray and capillary blood measurements (C-reactive protein, haemoglobin and blood glucose) could be performed. The standard clinical approach at the OAEOC offers hs-cTnT measurements to patients considered in need of further tests to rule-out AMI, but without the need of immediate hospitalisation (online supplemental table 1). The decision is left to the discretion of the treating GP.

Blood samples for the analyses of hs-cTnT were drawn from all included patients at 0,1 and 4 hours. Only the first hs-cTnT was considered in this subanalysis. Presenting symptoms, risk factors and time variables were registered at a predefined study form (online supplemental table 2). The upper reference limit (URL) for hs-cTnT (Elecsys Troponin T hs STAT assay, Roche Diagnostics, Switzerland) was $14 \mathrm{ng} / \mathrm{L}$ with a coefficient of variation of $<10 \%$, limit of detection (LoD) of $5 \mathrm{ng} / \mathrm{L}$ and limit of blank of $3 \mathrm{ng} / \mathrm{L}$ (additional details in the online supplemental appendix). ${ }^{23}$

\section{Single hs-cTnT strategy}

According to the ESC 0/1 hour algorithm for hs-cTnT, ${ }^{2}$ the single hs-cTnT rule-out strategy applies for patients where the first hs-cTnT is $<5 \mathrm{ng} / \mathrm{L}$, sampled 3 hours or more after symptom onset. A patient is triaged towards direct rule-in if the initial hs-cTnT is $\geq 52 \mathrm{ng} / \mathrm{L}$. Patients with values between these thresholds remain in the observation group in need of repeated hs-cTnT measurements. Following the guidelines, the troponin result should always be interpreted in conjunction with the clinical assessment and the ECG. ${ }^{2}$

\section{HEART score}

The original HEART score stratifies the risk for a major adverse cardiac event (MACE) during the first 6 weeks following presentation to an ED with symptoms suggestive of ACS. ${ }^{11} 12$ Each of the five components (History, ECG, Age, Risk factors and Troponin) provide a score of 0-2 points (table 1). Patients with a HEART score of 0-3 points (low risk) are considered suitable for rapid discharge, a score of 4-6 points (intermediate risk) signifies the need of further observation and patients with 7-10 points (high risk) are recommended early invasive strategies. ${ }^{11} 12$

In this study, the subjective History component was based on the presenting composition of non-typical and typical symptoms of ACS, ${ }^{11}$ as defined by the study investigators (online supplemental table 3). Only typical elements scored 2 points, a combination of typical and non-typical scored 1 point and only non-typical scored 0 points. The ECG component was calculated using the ECG obtained and interpreted by the GP at presentation. ECG with ischaemic ST-segment depression scored 2 points, non-specific changes in either the ST-segment, 


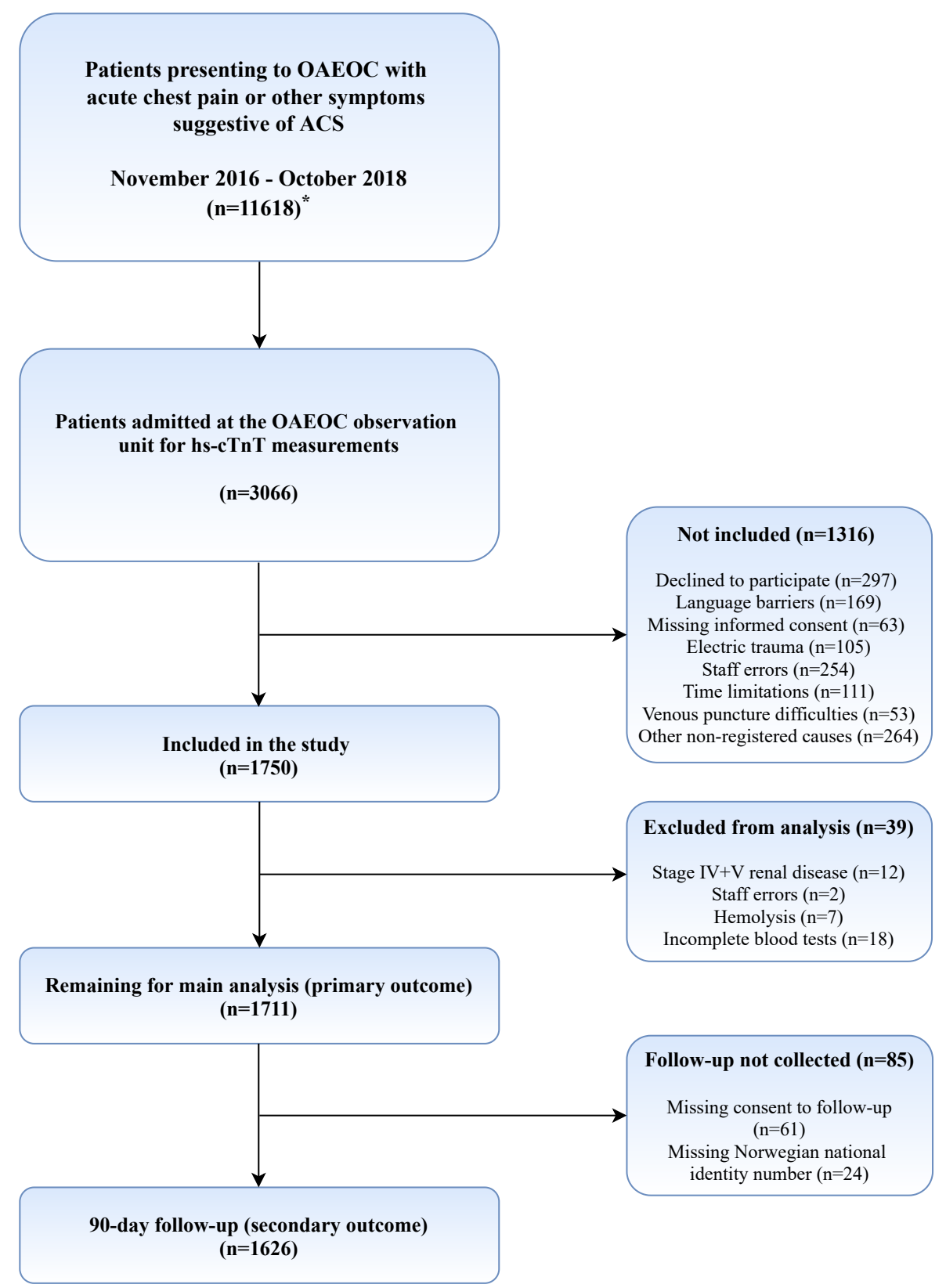

Figure 1 Patient flow chart. Study enrolment at the primary care emergency clinic during the OUT-ACS study. ${ }^{*}$ Critically ill patients are brought directly to hospital by the ambulance services. ACS, acute coronary syndrome; hs-cTnT, high-sensitivity cardiac troponin T; OAEOC, Oslo Accident and Emergency Outpatient Clinic; OUT-ACS, One hoUr Troponin in a low-prevalence population of Acute Coronary Syndrome.

T-inversions, Q-waves or left/right bundle branch block of unknown clinical significance scored 1 point and an ECG interpreted as normal scored 0 points. Among the Risk factors, obesity (BMI >30) was not systematically recorded. For the Troponin component, the first hs-cTnT sampled at the clinic was used, regardless of the time interval from symptom onset to blood draw. Hs-c TnT $\leq 14$ $\mathrm{ng} / \mathrm{L}$ scored 0 points, hs-cTnT 15-41 ng/L 1 point and hs-cTnT $\geq 42 \mathrm{ng} / \mathrm{L} 2$ points.

\section{Modified HEART score}

Since the single hs-cTnT rule-out strategy is based on hs-cTnT $<5 \mathrm{ng} / \mathrm{L}$ and the original HEART score operates with hs-cTnT $\leq 14 \mathrm{ng} / \mathrm{L}$ as the lowest cut-off value, we modified the HEART score by altering the Troponin component to more sensitive hs-cTnT thresholds. Inspired by a recent published study using a modified HEART score, ${ }^{24}$ a hs-cTnT $<5 \mathrm{ng} / \mathrm{L}$ resulted in 0 points, 1 point for hs-cTnT between LoD and URL (5-14 ng/L), and 2 points for hs-cTnT >URL (>14 ng/L). The four first components (History, ECG, Age and Risk factors) remained unchanged. 


\begin{tabular}{lll}
\hline $\begin{array}{l}\text { Table 1 } \\
\text { pain }\end{array}$ & The original HEART score for patients with chest \\
\hline History & Highly suspicious for ACS & 2 points \\
& Moderately suspicious & 1 point \\
& Slightly or not suspicious & 0 points \\
ECG & Significant ST-depression & 2 points \\
& Non-specific changes* & 1 point \\
Age & Normal & 0 points \\
& $\geq 65$ years & 2 points \\
& $46-64$ years & 1 point \\
Risk factorst & $\leq 45$ years & 0 points \\
& One or two risk factors & 2 points \\
& No risk factors & 0 point \\
Troponin & $\geq 3 \times$ URL & 2 points \\
& $>1$ to $<3 \times$ URL & 1 point \\
& $\leq$ URL & 0 points \\
Total & Low risk & $0-3$ points \\
& Intermediate risk & $4-6$ points \\
& High risk & $7-10$ points \\
\hline
\end{tabular}

Reproduced after the original HEART score ${ }^{12}$ with permission from the authors.

*Left bundle branch block, left ventricular hypertrophy, repolarisation changes, pacemaker.

† Risk factors: hypertension, diabetes mellitus, current or history of smoking, hypercholesterolaemia, obesity $\left(\mathrm{BMI}>30 \mathrm{~kg} / \mathrm{m}^{2}\right)$ and family history of coronary artery disease.

ACS, acute coronary syndrome; BMI, body mass index; ECG, electrocardiogram; HEART, History, ECG, Age, Risk factors and Troponin; hs-cTnT, high-sensitivity cardiac troponin T; URL, upper reference limit.

The HEART scores were applied and calculated retrospectively. During the HEART score assessments, the study investigators were blinded to patient identity, final diagnosis and other information not part of the HEART calculations.

\section{Outcome measures}

The primary outcome was the diagnostic performance for the rule-out of AMI at the index event for the three different strategies (single hs-cTnT, original and the modified HEART score).

The composite of AMI or all-cause death at 90 days was the secondary outcome, as a measure of the prognostic performance of the three different rule-out strategies. The 90-day follow-up data were collected through linkage with the Norwegian Cardiovascular Disease Registry ${ }^{25}$ as previously described. ${ }^{19}$

\section{Final adjudication of AMI}

For patients discharged home from the OAEOC, the final diagnosis was made by the treating GP based on all available information, including clinical assessment, repeated ECGs, 0, 1 and 4 hours hs-cTnT and additional lab analyses. The absence of a $0-4$ hour hs-cTnT delta in accordance with the Third Universal Definition of Myocardial
Infarction(1) served as a reference standard for ruling out AMI, as previously specified. ${ }^{19}$ For the hospitalised patients, the final diagnosis was also based on the Third Universal Definition of Myocardial Infarction(1) and adjudicated by two independent cardiologists with access to all collected data from the index episode, including data from the OAEOC and hospital discharge documents. In $19 / 227$ of the cases, a third cardiologist was involved in solving disagreements.

\section{Statistical analysis}

Numbers were presented as frequencies and percentages, means and standard deviations (SDs), or medians and IQRs, as appropriate. Comparisons of baseline characteristics between the single rule-out approach and the two low-risk HEART groups were made using the Pearson $\chi^{2}$ test or the Fisher exact test for categorical variables, whereas the Kruskal-Wallis test was used when comparing continuous variables. We used two-sided hypothesis testing with a significance level set at $\alpha=0.05$. The sample size calculation for the main study has been described previously. ${ }^{19}$

The rule-out performance of the three strategies were assessed by calculating sensitivity and the negative predictive values (NPVs), with corresponding 95\% CIs (online supplemental table 4 ). In addition, the specificity and positive predictive values (PPVs) were estimated to assess the accuracy of the rule-in and the high-risk HEART groups, and likelihood ratios were estimated for all categories. The overall diagnostic performance of the three strategies was illustrated by the area under the ROC (receiver operating characteristics) curve (AUC). To visualise the large intermediate groups in need of further testing, we used the predefined cut-off values for each group. The AUCs were compared using the $95 \%$ CIs and the McNemar's test with the single hs-cTnT strategy as the referent.

IBM SPSS V.26.0 (SPSS, IBM, Armonk, New York, USA), and Stata V.16.0 (Stata Corp, College Station, Texas, USA.) were used in the calculations.

\section{Ethics}

The study was approved by the Regional Committee North for Medical and Health Research Ethics (no. 2016/1241) and the Oslo University Hospital Information Security and Privacy Office (no. 2016/13308). Participation was based on written, informed consent. The OUT-ACS study is registered at ClinicalTrials.gov (NCT02983123) and was conducted in accordance with the STARD (Standards for Reporting Diagnostic Accuracy Studies) guidelines. ${ }^{26}$

\section{Patient and public involvement}

The patients or the public were not involved in the design, conduct, reporting or dissemination plans of our research. However, we have involved users from our formalised 'patient-contributors-to-research' group established by the Medical Department of Oslo University Hospital. We received clear indications that a fast and reliable rule-out of AMI in patients with chest pain has a high 
priority among users (patients). These inputs helped in the design and interpretation of the study.

\section{RESULTS}

\section{Study participants}

During the OUT-ACS study enrolment period (November 2016-October 2018), 3066 patients were transferred to the OAEOC observation unit for hs-cTnT measurements, with 1750 patients included in the study (figure 1). Thirty-nine patients were excluded from the primary analysis (figure 1 ), yielding 1711 participants. ${ }^{19}$ For $1529(89.4 \%)$ of the patients, the first hs-cTnT was measured 3 hours or more after symptom onset (table 2). The median time from OAEOC presentation to first blood sample was $136 \mathrm{~min}$ (IQR 100-194).

\section{Triage and baseline characteristics}

After a single hs-cTnT measurement, 569 (33.3\%) patients were assigned towards rule-out, 1098 (64.2\%) to the observation group in need of further hs-cTnT measurements, while $44(2.6 \%)$ patients were assigned towards rule-in. The original HEART score categorised $871(50.9 \%)$ of 1711 patients as low risk, $760(44.4 \%)$ as intermediate risk and $80(4.7 \%)$ as high risk. By applying the modified HEART score with more sensitive troponin thresholds, $639(37.3 \%)$ patients were triaged towards low risk, $876(51.2 \%)$ as intermediate risk and $196(11.5 \%)$ towards the high-risk group. Baseline characteristics of the single hs-cTnT rule-out and the lowrisk HEART groups are shown in table 2.

\section{Diagnostic and prognostic performance}

AMI was diagnosed in $61(3.6 \%)$ of 1711 patients at the index observation. Among the 569 patients directly ruled-out by the single hs-cTnT strategy, there were no incidents of AMI during the index episode. Hence, this approach had a rule-out sensitivity and NPV of $100.0 \%$ (figure 2 and table 3). Thirty-two (5.6\%) of the direct rule-out patients were hospitalised for other non-cardiac causes, whereas the remaining patients were discharged home (online supplemental table 5).

The HEART score risk-stratified more patients towards low risk $(\mathrm{n}=871)$ but missed five AMIs $(0.6 \%)$ during the index episode (details in online supplemental table 6$)$. This gives a sensitivity of $91.8 \%(95 \%$ CI $81.9 \%$ to $97.3 \%$ ) and NPV $99.4 \%$ (95\% CI $98.7 \%$ to $99.8 \%$, figure 2 and table 3 ). By applying the modified HEART score, only one patient with AMI $(0.2 \%)$ was misclassified as low risk $(\mathrm{n}=639)$, improving sensitivity to $98.4 \%$ (95\% CI $91.2 \%$ to $100.0 \%$ ) and NPV to $99.8 \%$ (95\% CI $98.9 \%$ to $100.0 \%)$. The number of low-risk patients in need of hospitalisation was $66(7.6 \%)$ and $40(6.3 \%)$ in the original and modified HEART groups (online supplemental table 5).

The PPV in the single rule-in and the original and modified high-risk HEART scores were $77.3 \%$ (95\% CI $63.8 \%$ to $86.8 \%$ ), $22.5 \%$ (95\% CI $15.5 \%$ to $31.5 \%$ ) and
$10.7 \%$ (95\% CI $7.6 \%$ to $14.9 \%$ ), respectively (table 3 ). In the single hs-cTnT rule-out group, one patient was considered high risk by both HEART scores (online supplemental table 7). The original and modified HEART score means were 3.6 (SD 1.6) and 4.2 (SD 1.8) points, respectively (online supplemental table 8).

The corresponding overall diagnostic performance, illustrated by the AUC for the three different strategies, was 0.85 (95\% CI 0.81 to 0.89 ), 0.77 (95\% CI 0.73 to 0.82 ), and 0.74 (95\% CI 0.70 to 0.78 ) (figure 3 ). Using McNemar's two-sided test, the results demonstrated that the single hs-cTnT strategy performed better than the original and modified HEART scores $(p<0.01)$.

The prognostic performance of the three rule-out approaches, as demonstrated by the composite of AMI or all-cause death at 90 days, was $0.0 \%$ for the single rule-out strategy and $0.7 \%$ and $0.2 \%$ for the original and modified low-risk HEART groups (figure 2 and table 3).

\section{DISCUSSION}

In the current secondary analysis of the OUT-ACS study, the single rule-out approach (hs-cTnT $<5 \mathrm{ng} / \mathrm{L}$ in patients presenting with symptom onset $\geq 3$ hours), had an excellent diagnostic and prognostic safety in a primary care emergency setting, with one-third of the participants triaged towards direct rule-out. Both the sensitivity and the NPV were $100.0 \%$, with no incidents of AMI or death for the following 90 days.

The original low-risk HEART score was less safe than the single rule-out strategy. Even though more patients were triaged towards low risk and early discharge, this entailed missing five AMIs, which we consider unacceptable. The sensitivity and the NPV in the low-risk HEART group improved with the modified HEART score, with only one missing AMI. On the other hand, the modified high-risk group had more false positives than the single rule-in group, which would have contributed to more hospitalisations. Similar data were found in an ED cohort, where patients with a modified HEART score $>3$ points had a lower risk for MACE than the original HEART score using a conventional troponin assay. ${ }^{27}$ All aspects considered, in our study, the simple single troponin approach was superior to both HEART scores when applied in a primary care emergency setting. In a recent hospital cohort, similar rule-out sensitivity was found after 6 weeks for the low-risk HEART group and the hs-cTnT $<$ LoD strategy. ${ }^{28}$ Although 6-week MACE was not reported in our study, several studies from the ED setting are in line with our results. One study demonstrated higher rule-out sensitivity for the hs-cTnI-only $(<3 \mathrm{ng} / \mathrm{L})$ strategy when compared with the modified low-risk HEART group. ${ }^{24}$ In a 1 year low-risk cohort, the safety of the hs-cTnT $<$ LoD strategy was not improved by the HEART score in late presenters (chest pain onset $\geq 3$ hours) ${ }^{29}$ And even though a direct comparison is not possible, our findings are somewhat consistent with a 
Table 2 Baseline characteristics

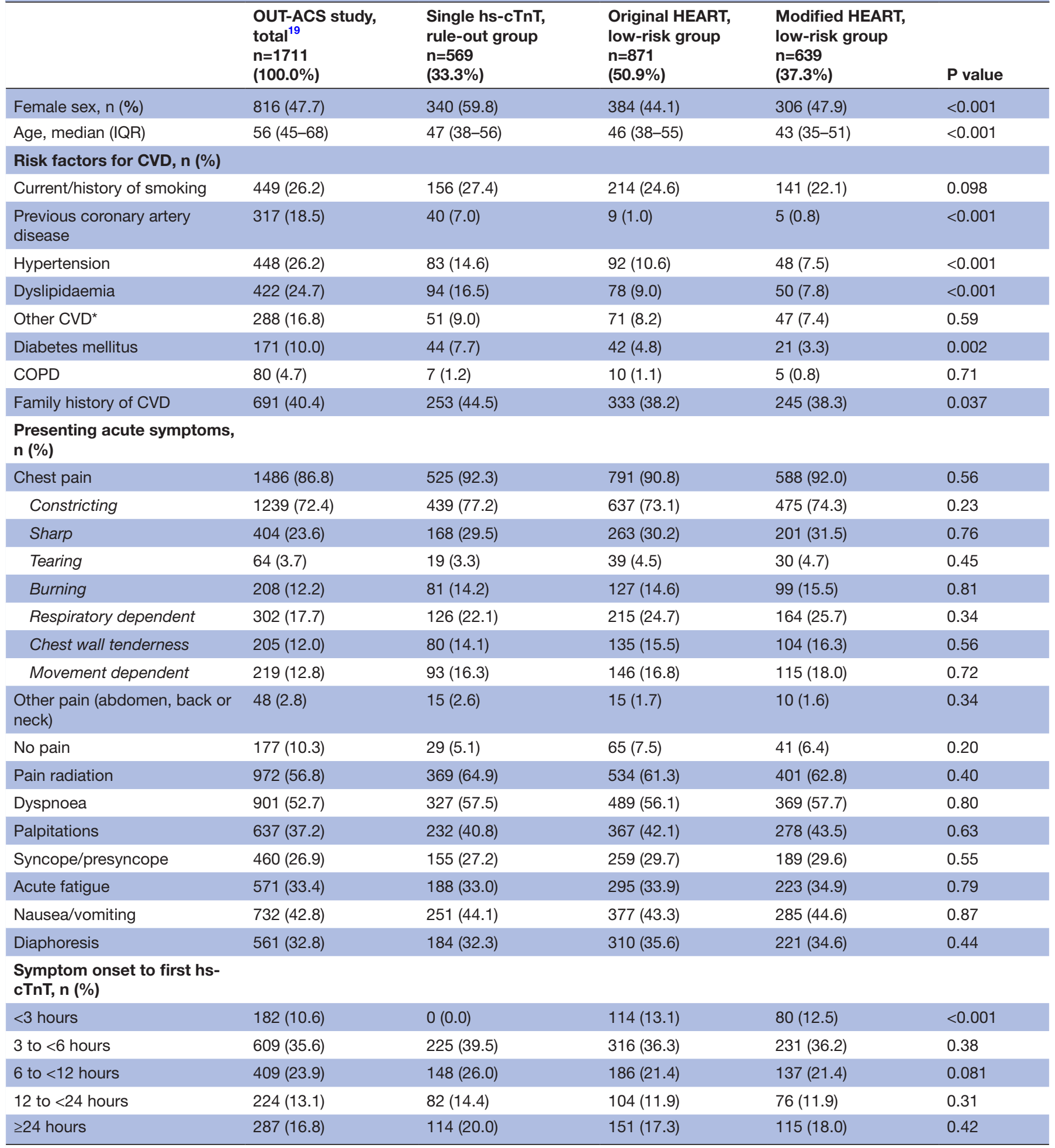

All values are presented as $\mathrm{n}(\%)$ and median (IQR). $\mathrm{P}$ values are calculated for comparisons across all three groups (single rule-out and the two low-risk HEART groups). The Pearson $\chi^{2}$ test or the Fisher exact test were used for the categorical variables and the Kruskal-Wallis test for the continuous variables.

*Includes atrial fibrillation, other arrhythmias, cardiomyopathies, cerebral stroke, heart failure or valvular disease.

COPD, chronic obstructive pulmonary disease; CVD, cardiovascular disease; HEART, History, ECG, Age, Risk factors and Troponin; hs-cTnT, highsensitivity cardiac troponin T; OAEOC, Oslo Accident and Emergency Outpatient Clinic.

previous study from the High-STEACS (High-Sensitivity Troponin in the Evaluation of Patients with Acute Coronary Syndrome) investigators, where clinical risk scores did not enhance the diagnostic rule-out performance when lower cut-off values for high-sensitivity cardiac troponin I (hs-cTnI) were applied. ${ }^{30}$ 


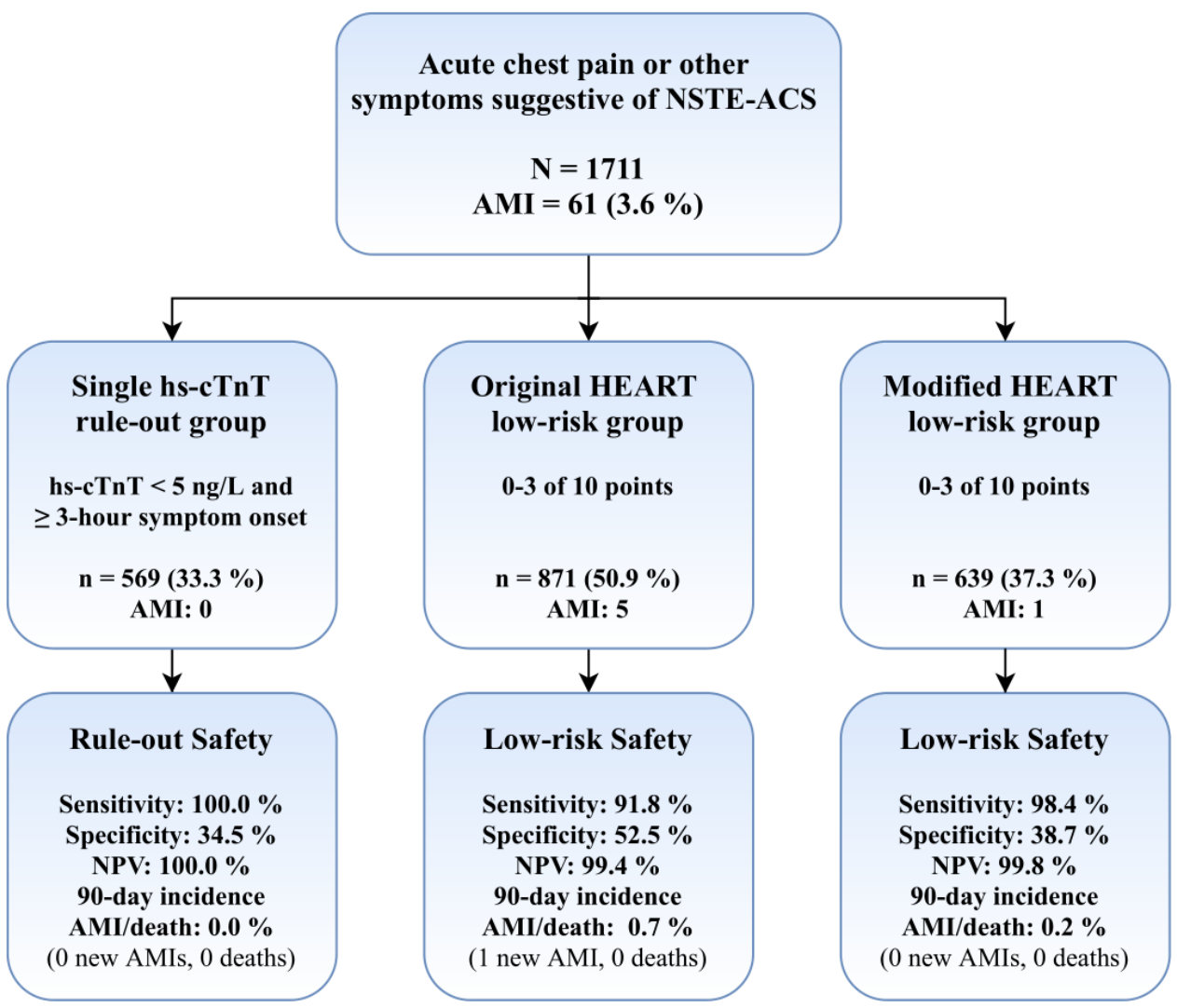

Figure 2 The diagnostic and prognostic safety of the three rule-out strategies at the primary care emergency clinic. AMI, acute myocardial infarction; HEART, History, ECG, Age, Risk factors and Troponin; hs-cTnT, high-sensitivity cardiac troponin T; NPV, negative predictive value; NSTE-ACS, non-ST-segment elevation acute coronary syndrome.

Our evaluation of the HEART score outside of hospitals differs from the previous EMS studies ${ }^{13} 14$ by not including patients with a highly suspected ACS. This is reflected by the low mean of both the original and the modified HEART scores (online supplemental table 8 ). Our study population comprised patients with lowto-intermediate risk in need of additional tests for a safe AMI rule-out, but not considered in urgent need of hospitalisation. This is also apparent from the delay in time to first hs-cTnT measurement, as the low-risk patients were rarely prioritised for rapid initial assessment. Our study also included patients with atypical symptoms such as acute fatigue, diaphoresis and acute dyspnoea without chest pain. The study was embedded in the regular clinical practice at the primary care clinic, which might increase both the external and internal validity of the results.

A recent cost-benefit analysis from the Netherlands demonstrated that patients categorised as low-risk HEART incurred high hospital expenses with limited benefits for each patient. ${ }^{31}$ Performing the initial hs-cTn workup of low-risk patients outside of hospitals might reduce unnecessary hospital admissions, healthcare utilisation and costs. Further implementation and cost-benefit studies in primary care are warranted.

Prehospital studies among paramedics have demonstrated that POC troponin assays should not be used to rule-out AMI due to low sensitivity. ${ }^{9}{ }^{10}$ However, the newer POC devices might perform better if tested at primary care emergency clinics, where they would be less subject to movement and temperature alterations. The novel hs-cTnI POC assays with diagnostic performance comparable to central lab assays may also show themselves valuable decision aids in primary care in the future. ${ }^{1718}$

Some limitations need to be addressed: first, only 61 $(3.6 \%)$ of 1711 patients in the OUT-ACS study were diagnosed with an AMI. Hence, the calculations on the diagnostic performance are based on few events and should be interpreted with care.

Second, the AMI diagnoses might be subject to verification bias, as the adjudication committee only evaluated hospitalised patients. For all patients discharged home, the final diagnosis was made by the discharging GP at the OAEOC. It would not have been ethical or feasible to admit all 1711 patients to the hospital for a similar diagnostic workup.

Third, there is no current consensus on how the subjective History component in the HEART score should be assessed. ${ }^{32}$ We based this component on the presenting symptoms registered at index and retrospectively categorised them as typical or non-typical for NSTE-ACS, as defined in online supplemental table 3.

Fourth, by assessing the HEART score retrospectively, the Risk factor 'obesity' was missing for all participants, as body mass index (BMI) was not systematically reported. 


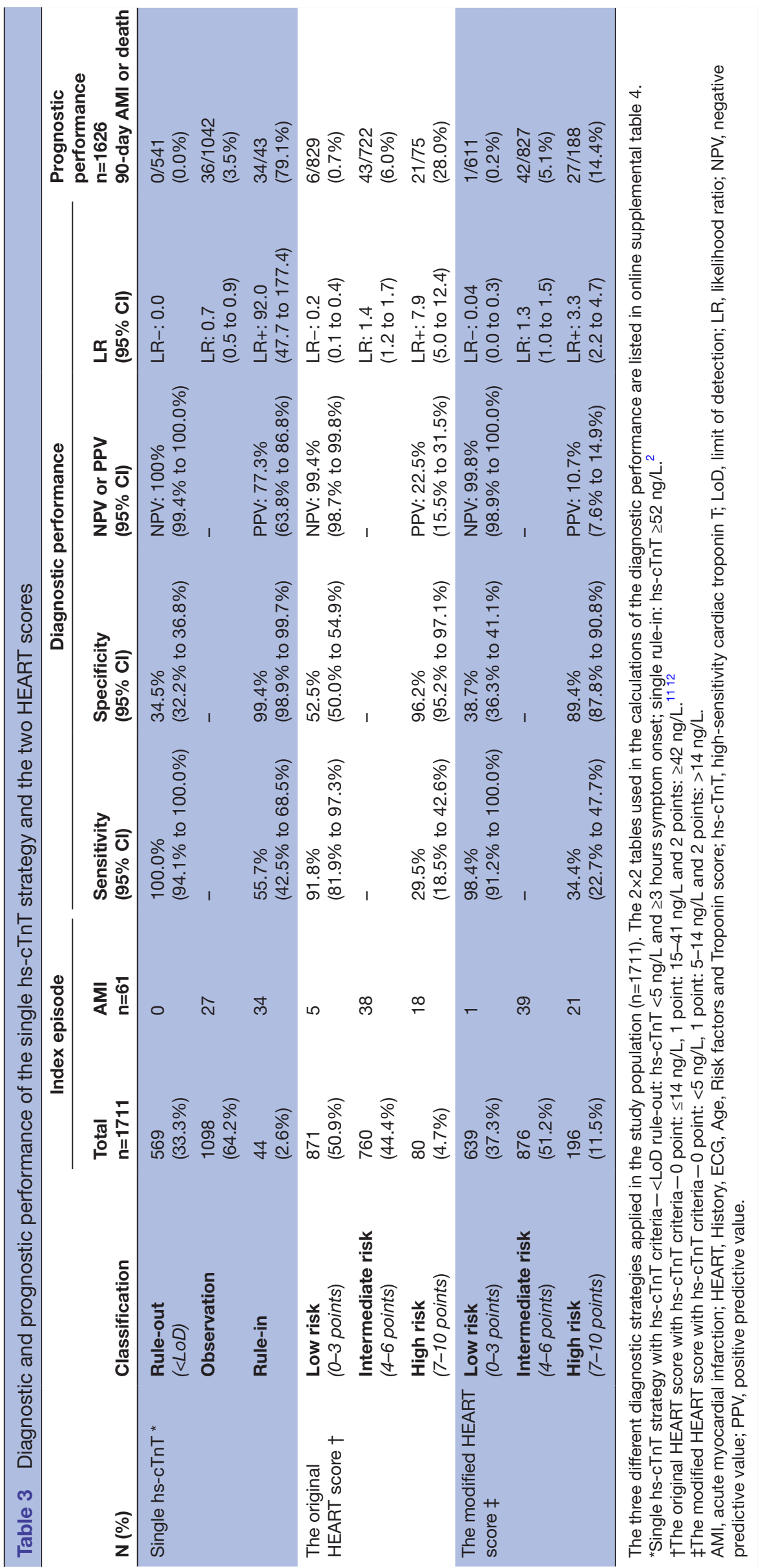




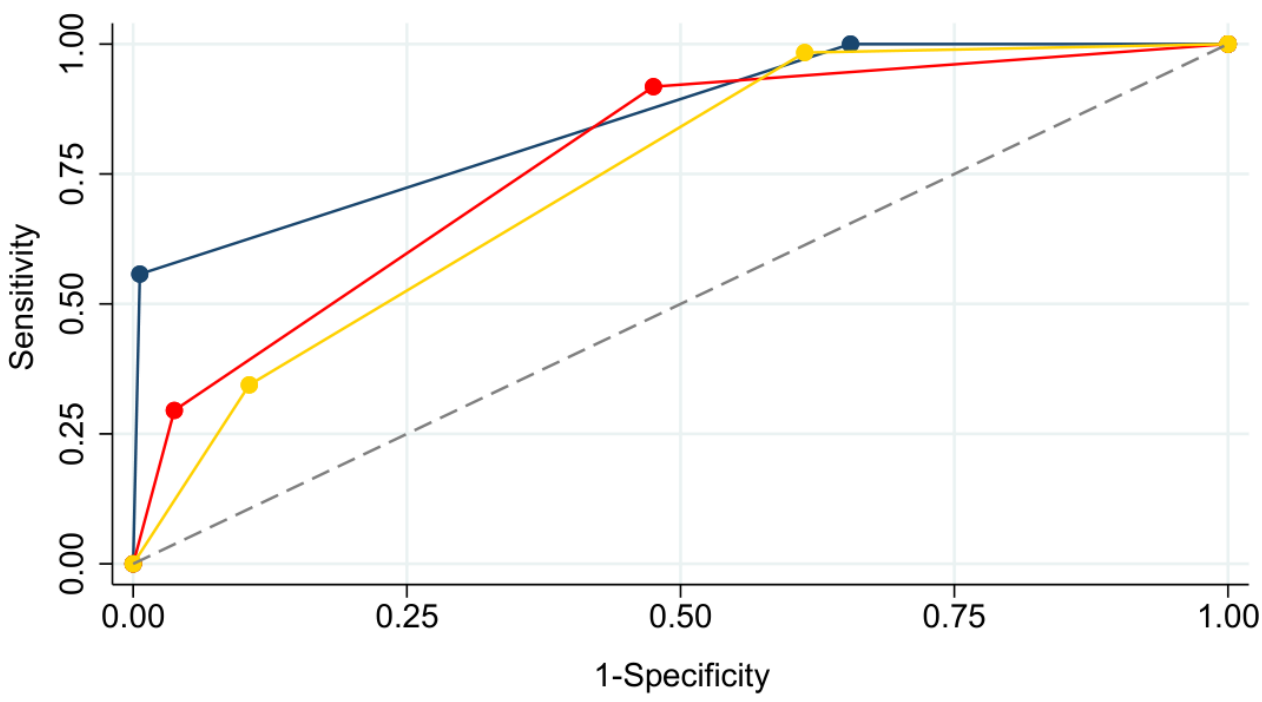

$\begin{array}{|lll|}\longrightarrow-\text { Single hs-cTnT } & \text { AUC: } 0.85 & (95 \% \mathrm{Cl} 0.81-0.89) \\ - \text { Original HEART score } & \text { AUC: } 0.77 & (95 \% \mathrm{Cl} 0.73-0.82) \\ -\begin{array}{l}\text { Modified HEART score } \\ --- \text { Reference }\end{array} & \text { AUC: } 0.74 & (95 \% \mathrm{Cl} 0.70-0.78) \\ & & \end{array}$

Figure 3 The overall diagnostic performance of the single hs-cTnT strategy, the original HEART score and the modified HEART score were illustrated by the AUC, which was achieved using the predefined cut-off values for the three different strategies. The single hs-cTnT rule-in and high-risk HEART groups are shown in the lower-left corner, the single hs-cTnT rule-out and low-risk HEART groups in the upper right. The large intermediate groups represent the patients in need of further tests. AUC, area under the curve; HEART, History, ECG, Age, Risk factors and Troponin; hs-cTnT, high-sensitivity cardiac troponin T.

Finally, the T component was based on the first hs-cTnT sample collected, regardless of symptom onset. Two of the five missed AMIs in the original low-risk HEART group had a symptom onset less than 3 hours before blood draw (online supplemental table 6). By using 3 hour onset as a prerequisite before rapid discharge, the diagnostic performance would have been improved. The onset of symptoms should, therefore, be taken into consideration if implemented in clinical practice.

In conclusion, when applying a hs-cTnT assay, the single hs-cTnT strategy $(<5 \mathrm{ng} / \mathrm{L}$ with symptom onset $\geq 3$ hours) was superior to the original HEART score in ruling out AMI in a primary care emergency setting. The rule-out safety of the HEART score was improved when lower troponin thresholds were used but at the cost of a low PPV. The single hs-cTnT strategy might have a great potential for simplifying and accelerating the triage of patients presenting with acute non-specific AMI symptoms in primary care, hence reducing unnecessary advanced testing, crowding in the EDs, and overall expenses.

\section{Author affiliations}

${ }^{1}$ Department of General Practice, University of Oslo, Oslo, Norway

${ }^{2}$ Department of Emergency General Practice, Oslo Accident and Emergency

Outpatient Clinic, Oslo, Norway

${ }^{3}$ Department of Cardiolgy, Oslo University Hospital, Ullevaal, Oslo, Norway

${ }^{4}$ Institute of Clinical Medicine, University of Oslo, Oslo, Norway

${ }^{5}$ Section of Cardiovascular and Renal Research, Oslo University Hospital, Ullevaal, Oslo, Norway
Twitter Tonje R Johannessen @tonjerj

Acknowledgements The authors would like to express their very great appreciation to the patients participating in this study, the staff at the observation unit at the OAEOC and the laboratory technicians at Oslo University Hospital, Ullevaal. The authors also wish to acknowledge the contributions provided by $\mathrm{Dr}$ Arne Westheim, Dr Barbra Backus, Dr Anne Kathrine Nore, Adjoa A Badu Antwi, Tone Klette Boehler and Linda Andersen. We are grateful for the support from the Norwegian Cardiovascular Disease Registry and the TSD facilities at the University of Oslo, IT Department (USIT).

Contributors TRJ, OMV, DA and SH contributed in the conception and design of the study. TRJ supervised the data collection and collated the data. TRJ and IM did the statistical analyses and analysed the data with contributions from OMV, SH and DA. ACKL, DA and SH contributed in the interpretation of the laboratory results. TRJ drafted the manuscript, and all authors contributed in revising and approving the final version for publication.

Funding Johannessen received funding from the Norwegian Research Fund for General Practice, the Norwegian Committee on Research in General Practice and the Norwegian Medical Association's Fund for Quality Improvement and Patient Safety.

Disclaimer The funders of the study had no role in study design, data collection, data analysis, data interpretation or writing of the manuscript.

Competing interests None declared.

Patient consent for publication Not required.

Provenance and peer review Not commissioned; externally peer reviewed. Data availability statement Data are available upon reasonable request.

Supplemental material This content has been supplied by the author(s). It has not been vetted by BMJ Publishing Group Limited (BMJ) and may not have been peer-reviewed. Any opinions or recommendations discussed are solely those of the author(s) and are not endorsed by BMJ. BMJ disclaims all liability and responsibility arising from any reliance placed on the content. Where the content includes any translated material, BMJ does not warrant the accuracy and reliability of the translations (including but not limited to local regulations, clinical guidelines, 
terminology, drug names and drug dosages), and is not responsible for any error and/or omissions arising from translation and adaptation or otherwise.

Open access This is an open access article distributed in accordance with the Creative Commons Attribution Non Commercial (CC BY-NC 4.0) license, which permits others to distribute, remix, adapt, build upon this work non-commercially, and license their derivative works on different terms, provided the original work is properly cited, appropriate credit is given, any changes made indicated, and the use is non-commercial. See: http://creativecommons.org/licenses/by-nc/4.0/.

\section{ORCID iDs}

Tonje R Johannessen http://orcid.org/0000-0001-9368-1471

Dan Atar http://orcid.org/0000-0003-1513-8793

Odd Martin Vallersnes http://orcid.org/0000-0003-1213-392X

Anne Cecilie K Larstorp http://orcid.org/0000-0002-0223-9248

Ibrahimu Mdala http://orcid.org/0000-0002-5204-1934

Sigrun Halvorsen http://orcid.org/0000-0001-7561-7644

\section{REFERENCES}

1 Thygesen K, Alpert JS, Jaffe AS, et al. Third universal definition of myocardial infarction. J Am Coll Cardiol 2012;60:1581-98.

2 Collet J-P, Thiele H, Barbato E, et al. 2020 ESC guidelines for the management of acute coronary syndromes in patients presenting without persistent ST-segment elevation. Eur Heart J 2020:ehaa575.

3 Burman RA, Zakariassen E, Hunskaar S. Management of chest pain: a prospective study from Norwegian out-of-hours primary care. BMC Fam Pract 2014;15:51.

4 Hoorweg BB, Willemsen RT, Cleef LE, et al. Frequency of chest pain in primary care, diagnostic tests performed and final diagnoses. Heart 2017;103:1727-32.

5 Harskamp RE, Laeven SC, Himmelreich JC, et al. Chest pain in general practice: a systematic review of prediction rules. BMJ Open 2019;9:e027081.

6 Nilsson S, Andersson PO, Borgquist L, et al. Point-of-care troponin T testing in the management of patients with chest pain in the Swedish primary care. Int J Family Med 2013;2013:1-7.

7 Schols AMR, Stakenborg JPG, Dinant G-J, et al. Point-of-care testing in primary care patients with acute cardiopulmonary symptoms: a systematic review. Fam Pract 2018;35:4-12.

8 Rasmussen MB, Stengaard C, Sørensen JT, et al. Predictive value of routine point-of-care cardiac troponin $T$ measurement for prehospital diagnosis and risk-stratification in patients with suspected acute myocardial infarction. Eur Heart J Acute Cardiovasc Care 2019;8:299-308.

9 Stopyra JP, Snavely AC, Scheidler JF, et al. Point-of-Care troponin testing during ambulance transport to detect acute myocardial infarction. Prehospital Emergency Care 2020;24:751-9.

10 Alghamdi A, Alotaibi A, Alharbi M, et al. Diagnostic performance of prehospital point-of-care troponin tests to rule out acute myocardial infarction: a systematic review. Prehosp Disaster Med 2020;35:567-73.

11 Six AJ, Backus BE, Kelder JC. Chest pain in the emergency room: value of the heart score. Neth Heart $J$ 2008;16:191-6.

12 Backus BE, Six AJ, Kelder JC, et al. A prospective validation of the heart score for chest pain patients at the emergency department. Int J Cardiol 2013;168:2153-8.

13 Ishak M, Ali D, Fokkert MJ, et al. Fast assessment and management of chest pain patients without ST-elevation in the pre-hospital gateway (famous triage): ruling out a myocardial infarction at home with the modified heart score. Eur Heart $J$ Acute Cardiovasc Care 2018;7:102-10.

14 van Dongen DN, Tolsma RT, Fokkert MJ, et al. Pre-hospital risk assessment in suspected non-ST-elevation acute coronary syndrome: a prospective observational study. Eur Heart J Acute Cardiovasc Care 2020;9:5-12.

15 McCord J, Cabrera R, Lindahl B, et al. Prognostic utility of a modified heart score in chest pain patients in the emergency department. Circ Cardiovasc Qual Outcomes 2017;10.

16 Santi L, Farina G, Gramenzi A, et al. The heart score with highsensitive troponin $\mathrm{T}$ at presentation: ruling out patients with chest pain in the emergency room. Intern Emerg Med 2017;12:357-64.

17 Sörensen NA, Neumann JT, Ojeda F, et al. Diagnostic evaluation of a high-sensitivity troponin I point-of-care assay. Clin Chem 2019;65:1592-601.

18 Boeddinghaus J, Nestelberger T, Koechlin L, et al. Early diagnosis of myocardial infarction with point-of-care high-sensitivity cardiac troponin I. J Am Coll Cardiol 2020;75:1111-24.

19 Johannessen TR, Vallersnes OM, Halvorsen S, et al. Pre-hospital one-hour troponin in a low-prevalence population of acute coronary syndrome: OUT-ACS study. Open Heart 2020;7:e001296.

20 Marshall GA, Wijeratne NG, Thomas D. Should general practitioners order troponin tests? Med J Aust 2014;201:155-7.

21 Zhelev Z, Hyde C, Youngman E, et al. Diagnostic accuracy of single baseline measurement of Elecsys troponin T high-sensitive assay for diagnosis of acute myocardial infarction in emergency department: systematic review and meta-analysis. BMJ 2015;350:h15.

22 Pickering JW, Than MP, Cullen L, et al. Rapid rule-out of acute myocardial infarction with a single high-sensitivity cardiac troponin $\mathrm{T}$ measurement below the limit of detection: a collaborative metaanalysis. Ann Intern Med 2017;166:715-24.

23 Giannitsis E, Kurz K, Hallermayer K, et al. Analytical validation of a high-sensitivity cardiac troponin T assay. Clin Chem 2010;56:254-61.

24 Body R, Morris N, Reynard C, et al. Comparison of four decision aids for the early diagnosis of acute coronary syndromes in the emergency department. Emerg Med J 2020;37:8-13.

25 Norwegian Institute of Public Health. Norwegian Cardiovascular Disease Registry, 2012. Available: https://www.fhi.no/en/hn/healthregistries/cardiovascular-disease-registry/ [Accessed 23 Mar 2020].

26 Bossuyt PM, Reitsma JB, Bruns DE, et al. STARD 2015: an updated list of essential items for reporting diagnostic accuracy studies. BMJ 2015;351:h5527.

27 Mark DG, Huang J, Kennedy CJ, et al. 60-day major adverse cardiac events in emergency department patients with non-low modified heart scores. Am J Emerg Med 2020;38:2760.e5-2760.e8.

28 Chew PG, Frost F, Mullen L, et al. A direct comparison of decision rules for early discharge of suspected acute coronary syndromes in the era of high sensitivity troponin. Eur Heart J Acute Cardiovasc Care 2019;8:421-31.

29 Sanchis J, Valero E, García Blas S, et al. Undetectable highsensitivity troponin in combination with clinical assessment for risk stratification of patients with chest pain and normal troponin at hospital arrival. Eur Heart J Acute Cardiovasc Care 2020;9:567-75.

30 Chapman AR, Hesse K, Andrews J, et al. High-sensitivity cardiac troponin I and clinical risk scores in patients with suspected acute coronary syndrome. Circulation 2018;138:1654-65.

31 van Dongen DN, Ottervanger JP, Tolsma R, et al. In-hospital healthcare utilization, outcomes, and costs in Pre-HospitalAdjudicated low-risk chest-pain patients. Appl Health Econ Health Policy 2019;17:875-82.

32 Marchick MR, Setteducato ML, Revenis JJ, et al. Comparison of 3 symptom classification methods to standardize the history component of the heart score. Crit Pathw Cardiol 2017;16:102-4 